\title{
Jean de Bueil, Le Jouvencel
}

\section{Maria Colombo Timelli}

\section{(2) OpenEdition \\ Journals}

\section{Édition électronique}

URL : https://journals.openedition.org/studifrancesi/20932

DOI : 10.4000/studifrancesi.20932

ISSN : 2427-5856

\section{Éditeur}

Rosenberg \& Sellier

\section{Édition imprimée}

Date de publication : 1 décembre 2019

Pagination : 554-555

ISSN : 0039-2944

\section{Référence électronique}

Maria Colombo Timelli, « Jean de Bueil, Le Jouvencel », Studi Francesi [En ligne], 189 (LXIII | III) | 2019,

mis en ligne le 01 mars 2020, consulté le 11 novembre 2021. URL : http://journals.openedition.org/ studifrancesi/20932 ; DOI : https://doi.org/10.4000/studifrancesi.20932

\section{Ce document a été généré automatiquement le 11 novembre 2021.}

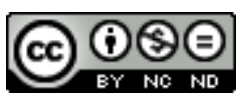

Studi Francesi è distribuita con Licenza Creative Commons Attribuzione - Non commerciale - Non opere derivate 4.0 Internazionale. 


\title{
Jean de Bueil, Le Jouvencel
}

\author{
Maria Colombo Timelli
}

\section{RÉFÉRENCE}

Jean de Bueil, Le Jouvencel, suivi du Commentaire de G. Tringant. Édition critique par M. Szkilnik, Paris, Honoré Champion, 2018, «Classiques Français du Moyen Âge» 182, $754 \mathrm{pp}$.

1 Texte fort représentatif de la production en prose du Xve siècle, Le Jouvencel (vers 1460) méritait bien une édition critique: ce long récit, biographie d'un personnage dont l'identité se cache sous le surnom que lui a attribué son capitaine, n'était en effet accessible que dans l'édition établie en 1887 par Léon Lecestre pour la Société de l'Histoire de France, selon des critères aujourd'hui largement dépassés. Ce qui s'offre aux lecteurs comme un manuel de guerre illustré par la carrière d'un personnage aussi imaginaire qu'emblématique, est en effet une œuvre fascinante, bien que difficile à classer: le but affiché dans le prologue est certes éminemment didactique, mais le texte y est néanmoins présenté comme «ung petit traictié narratif», ce qui ouvre largement les portes à l'ambiguïté ( $c f$. l'article «traité» dans le DMF; le syntagme est traduit 'récit didactique' dans le glossaire, p. 665).

2 Comme souvent, les choses sont plus compliquées qu'elles n'en ont l'air à première vue, et un des mérites de l'Introduction de M.S. (pp.9-117) est de faire le point sur de nombreuses questions dont certaines n'ont pas encore trouvé de réponse assurée. La première concerne l'identité même de l'auteur: traditionnellement identifié avec Jean de Bueil (1406-1477), grand capitaine au service de Charles VII, celui-ci semble avoir eu recours à la collaboration de trois "serviteurs» lors de la rédaction; d'autre part, l'interprétation du texte lui-même a interpellé les contemporains, ce qui a provoqué une lecture «à clef» quelques années seulement après sa composition, par Guillaume Tringant, écuyer de Jean de Bueil et auteur de l'«exposicion» éditée ici en annexe.

3 Quoi qu'il en soit, le récit est bien une fiction, un «roman» (p. 14), divisé en trois parties de dimensions inégales: la première consacrée à la jeunesse du protagoniste et à sa 
décision de se lancer dans l'exercice de la guerre, la deuxième à sa carrière militaire et à ses exploits, la dernière à ses succès, si importants qu'ils lui permettent d'épouser la fille d'un roi. Tout en refusant la dimension «historique» du texte, M.S. souligne combien celui-ci est redevable à la réalité politique et militaire de son temps, à tel point qu'elle s'interroge sur l'absence de Jeanne d'Arc - ou tout au moins d'une présence féminine équivalente - de la narration (pp. 21-28), pour conclure que finalement la figure historique de la Pucelle est «détournée» dans le texte: ses «bonnes qualités» seraient ainsi attribuée au Jouvencel lui-même, ses défauts à la demoiselle de Grant Fort, personnage très négatif, complice d'un groupe de traîtres et de rebelles.

4 Le Jouvencel est un texte agréable à lire, nostalgique souvent, drôle parfois, où des personnages fictifs agissent dans un cadre tout à fait vraisemblable: le portrait de l'homme de guerre qui en ressort est certainement idéalisé, mais ne manque jamais de réalisme, les dialogues sont vivaces, et l'on comprend bien la fascination qu'il a pu exercer sur les lecteurs de son époque. Seize manuscrits nous sont parvenus, produits pour la plupart dans un laps de temps réduit, preuve d'un succès rapide; il s'agit souvent de copies luxueuses - sur parchemin, enluminées - ayant appartenu à quelques grands seigneurs du Xve siècle: Louis de Bruges (manuscrit $B$ ), Jacques d'Armagnac (C), Philippe de Clèves (L), Jean de Laval (V); des artisans et artistes bien connus ont aussi été impliqués dans leur facture, que ce soit pour la copie (Colard Mansion pour les manuscrits $B$ et $G$ ) ou pour l'illustration (le Maître d'Edouard IV pour B, Jean Fouquet pour $N$, un de ses disciples pour $H$ ). La fortune du texte explique aussi l'intérêt que lui ont porté les premiers éditeurs, Antoine Vérard au premier chef, qui publia Le Jouvencel dès 1494 (remarquons qu'une étude reste à faire sur le modèle de cet incunable); les éditions de Philippe Le Noir (1523 et 1530), puis d'Alain Lotrian (s.d., 1531?) prouvent que le texte trouvait encore un public dans la première moitié du $\mathrm{xvI}^{\mathrm{e}}$ siècle.

M.S. confirme la répartition des manuscrits en deux familles, sur la base du contenu, dont la plus tardive (quatre mss) propose un texte volontairement raccourci; à l'intérieur de la famille plus ancienne (douze mss), on peut établir des parentés ultérieures (pp. 82-87). Soulignons, comme le fait l'éditrice, l'intérêt de la table des titres, qui assume dans certaines copies des proportions inattendues: loin de se limiter à une annonce synthétique du contenu, les titres y donnent un véritable sommaire du texte, dont ils reprennent des phrases entières (pp. 88-89). Le choix du manuscrit de base (N: Wolfenbüttel, Herzog August Bibl., Blankenburg 137) s'appuie essentiellement sur la date (ca 1470) et sur la qualité du texte, alors que pour le Commentaire de Tringant - qui manque dans $N$ - M.S. a eu recours au manuscrit de l'Escorial $(K)$, sans doute copié sur le premier.

6 L'analyse linguistique (pp.91-98 pour N, 99-102 pour $K$ ) est un peu rapide; elle a néanmoins le mérite de prendre en compte la ponctuation des manuscrits et de souligner l'intérêt du lexique (pour $N$ ) et de la syntaxe (pour $K$ ), aspect celui-ci qui peut raisonnablement être attribué à Tringant. Le toilettage du texte n'a pas posé de problème majeur (pp. 103-105).

7 Le texte (pp. 123-471, dont les 22 premières sont occupées par la Table des titres) est édité avec soin. La ponctuation pourrait parfois être enrichie, notamment pour aider le lecteur à reconnaître rapidement certaines incises: «Et cela fait, je vous meneray jusques au pié de la tour [virgule] ce me semble [virgule] sans que on nous voye» p. 185; «... et ferons porter noz eschielles avecques nous et aussi tous les autres habillemens qui nous sont necessaires, tellement que [virgule] se Dieu plaist, nous arriverons en la 
grant lande environ dix heures au soir» (p. 188). Ailleurs, au contraire, elle gagnerait à être allégée, avec un emploi plus étendu de la ponctuation moyenne: «Si la place est puissante de gens, il y fault gros guet [virgule] comme vous savez, et [virgule] selon que le guet sera, il vous fault tendre plus de chaynes. [deux-points, plutôt] S'il y a grant guet, grans chaynes. [point-virgule] S'il y a petit guet, moins de chaynes» (p. 305). Mais il s'agit là, comme le savent bien les éditeurs de textes, de choix individuels et toujours discutables.

Quelques suggestions graphiques: normaliser s'en suit / s'ensuit (les deux graphies paraissent par ex. p. 148, respectivement dans le titre du premier chapitre et à la fin du premier paragraphe); souder $a$ donner dans «car il est bien divers et semble tresestrange a homme nourry mollement et en grant delices... juner le plus du temps et a donner cops et recevoir» (p. 150); de même, souder $a$ faire dans «ilz auront tousjours a faire de vous» (avoir affaire, 'avoir besoin', p.161); distinguer parquoy adverbe et par quoy prép. + pronom relatif («junoient leurs chevaulx le plus du temps, parquoy ilz estoient vains, chetifs et maigres» p. 150); même chose pour atant adverbe et a tant à + adv. («Mais atant ne se tint pas», p. 151); j'aurais aussi distingué pource que et pour ce («Pource je conseille qu'on les laisse entrer au couvert» pp. 158-159).

9 L'apparat critique en bas de page enregistre les rares amendements et quelques caractéristiques matérielles du manuscrit, entre autres la présence de signatures indiquant la suite des cahiers). Les notes (pp. 473-497) portent principalement sur des aspects historiques (pratiques militaires $\mathrm{du} \mathrm{xv}^{\mathrm{e}}$ siècle, allusion à des personnages réels), plus rarement sur des traits stylistiques ou proprement philologiques (présence de variantes significatives dans la tradition manuscrite). Le choix des variantes proposé aux pp. 499-638 vise à donner un aperçu des différences entre les deux familles; M.S. n'hésite pas, le cas échéant, à reproduire de longs passages lorsque les deux versions s'éloignent sensiblement l'une de l'autre.

Le Glossaire (pp. 639-667) fait une large place au vocabulaire militaire, ce qui rendra service tant aux lecteurs qu'aux lexicographes. On y relève quelques entorses à l'ordre alphabétique (acquest acteur acquesta, redigé recueil recueillir reduire, succession survenant survenue substentation) et des renvois parfois incomplets (dépouillement des cent premières pages de l'édition): s.v. apertises et barbe a barbe ajouter le renvoi au titre 18 de la Table; bateau apparaît aussi sous la forme basteau, p. 191; s.v. clause ajouter le titre 26 de la Table; s.v. pululer on ajoutera a pululé, p. 171. Quelques gloses ne semblent pas convenir à tous les contextes: ainsi 'soigner' pour habiller dans «ilz habillerent leurs tronsons et les chevilles a ceulx qui devoient escheller» (p. 191), ou 'bruit' pour noise dans «entre les pouvres s'esmeuvent noises et dissentions pour assez pou de chose» (p. 149). On pourrait aussi suggérer quelques intégrations: apprimé, 'opprimé', rare (DMF et Gdf; "quant on cuide avoir le bout de ses besongnes on est apprimés au commencement» p.175); curieux de, 'qui se préoccupe de, qui prend soin de' ([le corbeau] «devient curieux de leur [= de ses petits] gouvernement» p. 172; dittateurs, 'ceux qui rédigent ou dictent un texte, auteurs' («les anciens orateurs, dittateurs et historiens» p. 146); exaulcer, 'élever (en importance)' («Dieu ne le exaulça pas si hault que...» p. 167); finer, 'disposer de qqn' («il faut adviser quelles gens nous finerons et en quel nombre» p. 186); langaige, 'propos, discours' («pour donner fin a mon langaige» pp. 168-169); mais que, 'pourvu que, à condition que' («mais qu'il ne soit dommagé du corps» p. 180 et ailleurs); maleuretez, 'malheurs' («les maleuretez qu'on peut avoir a la guerre» p.169); obstant, prép., 'malgré’ («obstant l'eaue qui est ou fossé» p.178); 
pourquoy, subst., 'ce qui est nécessaire' («Qui veult acquerir bonne renommee il doit premierement faire le pourquoy» p.156); route, 'troupe, compagnie' («Ainsi ne demourerent que dix en la principale route» p. 159); ravoir, 'retrouver' (la forme rara risque de dérouter: «on le rara bien quoy qu'il couste» p. 180); sur, adv. («Sur, Jouvencel et vous, Pietres, desploiez voz basteaux» p. 191); travailler, 'voyager, chevaucher' («j'avais longuement travaillé et avoye besoing de repos» p. 149); voir, 'vrai', banal pour un spécialiste, mais ambigu en français moderne («pour voir vous dire» p. 148 et ailleurs); voise, subj. P3 de aller («quelque part qu'il voise» p. 134 et ailleurs). Parmi les locutions, on pourrait signaler: «la plus grande et saine opinion de son conseil» (p. 143: 'la plus grande partie, la quasi-totalité'); ou «ilz cuidoient qu'on les tenist ja par la queue» (p. 177: 'attraper qqn', seule occurrence dans DMF et dans Di Stefano 2015).

11 Le Glossaire est suivi de l'Index des noms propres (pp.669-686) et d'un relevé des proverbes et expressions gnomiques (pp.687-690); remarquons que l'usage fait par l'auteur des phrases sentencieuses mériterait une étude à part: si celles-ci se situent souvent en ouverture de chapitre - ce qui rentre en plein dans les habitudes médiévales - elles peuvent être évoquées encore en conclusion, ce qui paraît moins fréquent, afin de souligner la valeur exemplaire de l'épisode raconté. Un seul exemple: le chapitre 2 de la deuxième partie s'ouvre par «Il advient souvent que quant on cuide avoir le bout de ses besongnes on est apprimés au commancement. Et semblablement quant on cuide deceoir on est deceu. Pour ce dit on que l'omme propose et Dieu dispose...» (p. 175); et se termine par «Et c'est la fin de ce present chapitre, qui demonstre evidemment et mect en effect le commancement d'icelui» (p. 182).

12 Comme on l'a dit, M.S. complète l'édition du Jouvencel par celle de l'«exposicion» de Guillaume Tringant (intitulée ici Commentaire), elle aussi accompagnée de tous les apparats complémentaires: notes (pp.717-721), variantes des deux autres mss conservant le texte (pp. 723-731), glossaire (pp.733-735), index des noms propres (pp. 737-752, particulièrement riche en informations). Le but de Tringant est bien d'«exposer», c'est-à-dire 'expliquer, commenter', le récit de Jean de Bueil, ce qui attirera certainement l'attention des historiens, s'agissant d'un témoignage non seulement contemporain, mais surtout basé sur des sources de première main, l'Auteur se proclamant serviteur du Jouvencel au même titre des trois autres qui auraient collaboré à la rédaction du texte. On l'aura compris: l'intérêt du Jouvencel n'est pas qu'historique et culturel, et une bonne édition ne pourra qu'encourager la lecture d'un texte riche et fascinant, et fournir la base solide pour des études ultérieures, entre autres linguistiques. 